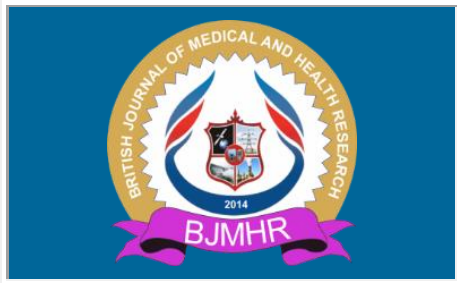

\title{
BJMHR
}

British Journal of Medical and Health Research

Journal home page: www.bjmhr.com

\section{Role of Immunity: Current Status of COVID-19-A Review}

\section{E Prabhakar Reddy ${ }^{1^{*}}$, T.Mohanalakshmi ${ }^{2}$ \\ 1. Professor of Biochemistry and Central Laboratory Head, Sri Lakshmi Naryana Institute of Medical Sciences, Puducherry, Affiliated to BIHER. \\ 2.Associate Professor of Microbiology, Sri Lakshmi Naryana Institute of Medical Sciences, Puducherry, Affiliated to BIHER.}

\section{ABSTRACT}

The immune system in a broad sense is a mechanism that allows a living organism to discriminate between "self" and "non-self." Corona viruses are members of a large family of viruses causing illnesses associated with respiratory tract. These viruses belonging collectively to the order Nidovirales, are positively Stranded RNA viruses. Respiratory virus infections have a major impact on health. Mostly Virus gains the ability to spread efficiently within a new host that was not previously exposed or susceptible. Acute respiratory illnesses, mostly caused by viruses, are the most common illness experienced by otherwise healthy children and adults worldwide. Humans may be infected by and suffer clinical consequences from numerous different viruses, and in most instances the infection is resolved with or without tissue damage. Reinfection is usually subclinical, and for many viruses we have effective vaccines. Therefore, it is of the utmost importance to understand the pathophysiology of disease and how the immune response to the pathogen affects the disease. Although the immune system plays an important role in fighting COVID-19, paradoxically it could also be harmful. Herd immunity is the indirect protection of individuals from an infectious disease when a high proportion of a population is immune (usually through vaccination). Persons who haven't been infected, or who haven't had a good immune response, are protected because there are enough immune people around them to slow or stop person-to-person transmission. There is currently insufficient information to conclude whether people who have recovered from COVID-19 and have antibodies are protected from a second infection. The development of vaccines is considered to be immunology's greatest success, as evidenced by the millions of lives saved. Boosting the immune system is a potential resource for the treatment of patients with COVID19. The process and mechanism of the immune system can be a good source of knowledge for immune system development. Finally, nutrition (eg, dietary recommendations) to boost the immune system should be explored and recommended.

Keywords: Immune system, Corona virus, Respiratory infection, Polymer Chain Reaction, Herd immunity

*Corresponding Author Email: drpebyreddy@yahoo.com Received 05 September 2020, Accepted 19 October 2020

Please cite this article as: Reddy EP et al., Role of Immunity: Current Status of COVID-19-A Review. British Journal of Medical and Health Research 2020. 


\section{INTRODUCTION}

Early immune system discoveries were largely fuelled by a desire to prevent the spread of disease and develop better treatments for the sick. As far back as the eighteenth century, microbiologists sought to inoculate healthy people against diseases. In fact, vaccines were created to combat illness before anyone could prove that microbes caused illness, or that immune cells could kill microbes. The immune system is amazingly complex. It can recognize and remember millions of different enemies, and it can produce secretions and cells to match up with and wipe out each one of them. Evolutionary factors that affect a pathogen's ability to infect a novel host. The emergence of human diseases from animal virus by switching hosts from animal to humans and was subsequently transmitted within human populations result in outbreak, mortality and economic burden. Newly emerging viral diseases are major threats to public health.

The immune system is the major protective system of the body against invading foreign bodies and infectious agents ${ }^{1}$. Human immune system is one of the most critical and complex systems responsible for mediating response against foreign agents ${ }^{2}$. Such system could be the inborn innate immunity involving group of cells much organized up to organ levels that serve to protect the body against any infection. Such innate immune system involves one of the first line of immune response ${ }^{3}$. The innate immune system comprises of organs including the skin responsible for the major protection of the body ${ }^{4}$. Continuously, it includes group of cells protecting the body against any foreign invading material without any further categorization ${ }^{4}$. These cells mostly ferocious in their actions kill or damage the invading foreign body, release chemicals that ultimately kills the foreign agent ${ }^{5}$. Adaptive immunity on the other hand which is also referred to as the acquired immunity is the other type of immune response ${ }^{6}$. It is mostly concerned with detailed and more specific action against invading microbes, toxins and even mediating immune response between donor and recipient during organ or tissue transplants ${ }^{7}$. It is much controlled and specified form of immunity and involves key mechanisms for activation of different cells. This acquired immunity could mediate a cellular based response or humoral immunity ${ }^{8}$. Viral infections have been associated with the type of the hosting system and nature of organs. Principally the nature of infection and mode of immune response could influence the pathogenesis of the disease ${ }^{9}$. Considering the respiratory system, it comprises the airways which are formed by a layer of epithelial cells which in essence is part of the innate immunity. Mucosal secretions by the epithelial wall serve also as part of the innate immunity. However, with a lot of viral population being part of the host microbiome particularly in the respiratory system, it may affect a possible antiviral immune response ${ }^{10}$. Similarly, various modifications during leucocytes differentiation might allow for lack of 
responses against the viral population in the microbiome. With that said however, no available studies have shown direct relation between onset of viral immune response and the microbiome in respiratory tract ${ }^{11}$.

In this Review describes up-to-date information on laboratory methods presently available in the diagnostic virology laboratories and also discuss our current understanding of the circumstances of Respiratory infection and Role of Immune system, Infectious disease Transmission and Current status of Vaccine and Herd Immunity. Understanding such issues could prove to be useful in the future for the control and perhaps prevention of infectious Diseases.

\section{DISCUSSION:}

Corona viruses are members of a large family of viruses causing illnesses associated with Respiratory tract. These viruses belonging collectively to the order Nidovirales, are positively Stranded RNA viruses ${ }^{12}$. The corona virinae are further subdivided into alpha, beta and gamma subfamilies each representing a particular nuclear configuration of the viral RNA ${ }^{13}$. The viral RNA contains a 5'cap and 3' poly A tail allowing to act as a messenger RNA for expression of its replication machinery proteins ${ }^{12}$. Thus a major part of the genome encodes for the replication machinery proteins hence its rapid replication upon entry into the host cell. Structural organization of the viral cell showed the virus as cylindrical with 4 specified proteins encoded by the minor sections of the genome ${ }^{14}$. These include the embrane (M) protein, the Spike (S) protein, Envelop (E) protein and Nucleocapsid(N) protein. A major distinction is seen in the beta subfamily where another (5th) structural protein is observed, the Hemagglutinin esterase protein ${ }^{12}$. The protein is thought to enhance spike protein activity and establish interaction with sialic acids on glycoproteins present on the surface of host cells there by facilitating viral entry ${ }^{15}$. Upon viral entry, the expression of the replication machinery is achieved. The Replicase protein expression is achieved in a series of complex mechanisms starting with expression of two polyproteins ppla and pplb ${ }^{12}$. Next is the assembly of the viral replication complex to replicate the Viral RNA.

The replication machinery utilizes viral RNA to synthesize the viral genome again. The viral RNA synthesis proceeds with formation of Genomic RNA and the sub genomic RNA which is concerned with expression of structural and accessory proteins. Their encoding genes are located along the 3' terminal end representing nest of RNA ${ }^{16}$. The process is completed with translation of the structural proteins of the virus which are then transferred to the Endoplasmic Reticulum, assembled and further released as mature various.

Its pathophysiology involves viral entry in respiratory droplets into the lungs via the airways 12. Major target of the virus is the alveolar cells which play critical roles in gaseous exchange 
during respiration ${ }^{17}$. Interaction between the viral Spike proteins and the ACE receptor ensures viral entry into the alveolar cells with the concomitant of structural disintegration. The Inflammatory response initiated was due to release of pro-inflammatory cytokines such as IL6 and TNF-alpha ${ }^{18}$. These two cytokines interact with the brain's hypothalamus resulting in physiological changes including increase in body temperature and fever. Additionally, the response results in vasodilation and increase vascular permeability. As such, with corresponding fluid accumulation, causing increased breathing capabilities and by extension breathing difficulty ${ }^{12}$. Researchers have continued to investigate the mechanism of antiviral response to the SARS-Cov2 and there have been positive results. Moreover, there is hope that in the near future, vaccine and definitive treatment to Covid-19 will come to fruition.

\section{Respiratory virus infections and Laboratory Tests:}

Respiratory virus infections have a major impact on health. Acute respiratory illnesses, mostly caused by viruses, are the most common illness experienced by otherwise healthy children and adults worldwide. Common respiratory viruses include influenza A and B, respiratory syncytial virus $A$ and $B$, parainfluenza virus types $1-3$, adenovirus, rhinovirus, human metapneumovirus, and coronavirus types OC43 and 229E. Less common respiratory viruses include parainfluenza virus type 4 , influenza virus $\mathrm{C}$, and specific types of enteroviruses. The significance of more recently discovered viruses such as human bocavirus, coronavirus NL63, and HKU1 has yet to be elucidated ${ }^{19-20}$.

Clinical presentations of respiratory virus infections overlap among those caused by various viruses. In addition, clinical manifestations may mimic those of diseases caused by bacteria. Therefore, antibiotics are most often used in these infections, most of them unnecessarily. Furthermore, LRIs often require hospitalization for management such as intravenous antibiotics and symptomatic and supportive treatment. Specific antiviral treatment for respiratory virus infections is only available for influenza. Respiratory viral diagnosis is an integral part of patient management. Accurate diagnosis of specific respiratory virus infection not only improves the knowledge of disease the patient has but also can affect patient management and help prevent secondary spread of the infection. Rapid viral diagnosis may result in discontinuation of unnecessary antibiotics, initiation of antiviral drug for influenza, reduction of costs related to reduction of unnecessary investigations, and shortened hospital stay $^{21-23}$.

Because of the sensitivity and rapid turn-around time, nucleic acid amplification technologies such as PCR will likely be the focus of further advances in respiratory virus diagnostics. The future will likely promise additional commercial test kits for molecular detection of respiratory viruses, including multiplexed assays. Because of their exquisite sensitivity, nucleic acid amplification tests can create diagnostic conundrums. Additional research is needed to 
elucidate the clinical significance of positive PCR results that are persistent in a patient, and when two or more respiratory viruses are detected in a single specimen.

Current and future diagnostic options will include antigen, molecular, and culture-based methods. The performance characteristics and limitations of these methods will vary greatly with the new generations of assays. It is important that diagnostic virologists and clinicians understand these characteristics and limitations.

\section{Immune system:}

Humans may be infected by and suffer clinical consequences from numerous different viruses, and in most instances the infection is resolved with or without tissue damage. Reinfection is usually subclinical, and for many viruses we have effective vaccines; classic examples include measles, mumps, rubella, rotavirus and varicella zoster viruses. Other viruses, such as HIV, hepatitis $\mathrm{C}$ virus (HCV), hepatitis B virus (HBV) and some herpesviruses, can cause substantial tissue damage in some or all individuals they infect, and lesions can become chronic. These viruses usually have one or more properties that allow them to diminish the efficacy of host adaptive or innate immunity, and we lack effective vaccines against most of these agents. Infection with viruses such as influenza virus and respiratory syncytial virus (RSV) has a variable outcome. Most individuals may suffer mild or subclinical infection, but others experience severe disease that can be lethal.

When the immune system is missing one or more of its components, the result is an immunodeficiency disorder. Immunodeficiency disorders can be inherited, acquired through infection, or produced unintentionally by drugs such as those used to treat people with cancer or those who have received transplants. Temporary immune deficiencies can develop in the wake of common virus infections, including influenza, infectious mononucleosis, and measles. Immune responses can also be depressed by blood transfusions, surgery, malnutrition, smoking, and stress. Some children are born with poorly functioning immune systems. Some have flaws in the B cell system and cannot produce antibodies. Others, whose thymus is either missing or small and abnormal, lack T cells. Very rarely, infants are born lacking all of the major immune defenses. This condition is known as severe combined immunodeficiency disease or SCID.

Scientists are now able to mass-produce immune cell secretions, both antibodies and lymphokines, as well as specialized immune cells. The ready supply of these materials not only has revolutionized the study of the immune system itself but also has had an enormous impact on medicine. Scientists have learned much about the immune system, they continue to study how the body launches attacks that destroy invading microbes, infected cells, and tumors while ignoring healthy tissues. New technologies for identifying individual immune cells are now letting scientists quickly determine which targets are triggering an immune response. The 
combination of new technology and expanded genetic information will no doubt teach us even more about how the body protects itself from disease.

Coronaviruses (CoVs), mainly targeting human respiratory system, are responsible for healththreatening outbreaks including severe acute respiratory syndrome (SARS), Middle East respiratory syndrome (MERS) and lastly coronavirus disease 2019 (COVID-19) ${ }^{24}$. In December 2019, in the Chinese Province of Wuhan the novel coronavirus has been identified in patients with atypical pneumonia characterized by fever, dry cough and progressive dyspnea 25. Rapidly, this coronavirus, namely SARS-CoV-21, has spread worldwide, leading to a serious lung inflammation, acute respiratory distress syndrome (ARDS), cardiac and renal injury, especially in patients with older age and comorbidities (diabetes mellitus, hypertension, and heart failure) ${ }^{26-28}$.

It has been suggested that during the response to SARS-CoV-2, the immune dysregulation and the high level of proinflammatory cytokines could be the main cause of tissue injury. Eventually, the exact pathophysiologic mechanism of COVID-19 remains still largely unknown. The effective antiviral responses of the host innate and adaptive immunity, including the production of various proinflammatory cytokines, the activation of T cells, CD4 and CD8+ $\mathrm{T}$ cells, are essential for controlling the viral replication, limiting the spread of virus, inflammation and cleaning the infected cells ${ }^{29-30}$. Nevertheless, the tissue injury caused by the virus could induce the exaggerated production of proinflammatory cytokines, the recruitment of proinflammatory macrophages and granulocytes. This results in the cytokine storm (CS) termed as a macrophage activation syndrome (MAS) or secondary hemophagocytic lymphohistiocytosis (sHLH), thus leading to further tissue damage ${ }^{31-33}$.

Data from recent studies have suggested that SARS-CoV-2 infection can lead to immune dysregulation through affecting the subsets of $\mathrm{T}$ cells. The significant alleviation of $\mathrm{T}$ cells is observed in COVID-19 and more pronounced in severe cases. In patients with COVID-19, the level of helper T cells (CD3+, CD4+) and cytotoxic suppressor T cells (CD3+, CD8+), and regulatory $\mathrm{T}$ cells are below normal level while helper $\mathrm{T}$ cells and regulatory $\mathrm{T}$ cells in severe patients are remarkably lower than non severe patients. Regulatory $\mathrm{T}$ cells are responsible for the maintenance of the immune homeostasis with suppressing the activation, proliferation, and proinflammatory function of most lymphocytes including $\mathrm{CD}+4 \mathrm{~T}$ cells, $\mathrm{CD}+8 \mathrm{~T}$ cells, NK cells, and B cells ${ }^{34-35}$.

In addition to $\mathrm{T}$ cells, the reduction of $\mathrm{B}$ cells and NK cells are seen in COVID-19. Another important result is the confirmed strong relationship between inflammatory markers, including ESR, CRP and IL-6 and the subset of lymphocytes ${ }^{36}$. However, previously it has been shown that there is no significant correlation between IL-6 and subsets of lymphocytes ${ }^{37}$. Although these reports have indicated that $\mathrm{CD}+4 / \mathrm{CD}+8 \mathrm{~T}$ cell ratio in SARS-CoV-2 infection is similar 
to the healthy group, the increase in this ratio and the decline of CD+8 T cells and B cells are considered as a poor predictor for the assessment of post-treatment clinical follow-up ${ }^{36-37}$.

Taken together, these results indicate that SARS-CoV-2 is responsible for an immune dysregulation with the induction of aberrant cytokine and chemokine response, alteration in level of the subgroup of lymphocytes all of which might result in cytokine storm and further tissue damage. Excessive inflammatory response with features of cytokine storm cause severe disease course and worsens the prognosis in COVID-19. Undoubtedly, definitive and most effective treatments for COVID-19 drugs would be the antiviral agents that directly target SARS-CoV-2.

\section{Infectious Disease transmission:}

Mostly Virus gains the ability to spread efficiently within a new host that was not previously exposed or susceptible. This may be achieved by either increased exposure or the gaining of variations that allow them to overcome barriers to infection of the new hosts, in these cases, outbreaks will be the results since that have the ability to spread efficiently between individuals in populations of the new host. Transferring successfully over great phylogenetic distances might be the reason for the novel SS positive sense RNA COVID-19 shift to human form unknown host believed to be the result of Zoonotic transmission. Following a host shift favours mutations that allow utilizing better cellular machinery, enhancing immune avoidance, replication, optimizing virulence, and maximizing the transmission potential might be the reason for pandemic outbreak. Overall 2, 45,648 cases were confirmed with COVID- 19 infection in which 191 cases from India and death rises to 10,048 of which 4 from India as on 19 March $2020{ }^{38}$. The percentage of death was greater in male gender (2.8\%) than female $(1.7 \%)$ this may be due to respiratory complications because of smoking. Further physically more active by male by which circulatory system cannot keep up with the available oxygen by the muscles. In order to maintain the energy level, muscles shift from aerobic to anaerobic metabolism by which break down of carbohydrates anaerobically to generate energy result in pyruvate metabolic products in the absence of oxygen converted into lactic acid. These modifications of the energy level utilization by the viral infected cells can increase available energy for viral replication ${ }^{39}$.

India is a developing country were most of the Government hospitals are well ventilated and more number of open market (Sandhai) are available around the country. Well-designed natural ventilation systems can often be more effective than air conditioning in promoting effective infection control, by increasing the number of air exchanges ${ }^{40}$. Poorly ventilated buildings affect air quality have higher risks of infectious disease transmission for patients, workers, and visitors might be the reason for high number of COVID-19 positive cases observed in most of the developed countries. 


\section{Current Status:}

The COVID-19 pandemic is an ongoing issue that affects the lives of most people around the world. Most countries are now semi-closed, strict travel regulations have been enacted, international relations have been affected, and humans are experiencing an unprecedented regime, which has changed ordinary life. Therefore, it is of the utmost importance to understand the pathophysiology of disease and how the immune response to the pathogen affects the disease. Although the immune system plays an important role in fighting COVID-19, paradoxically it could also be harmful.

Most COVID-19 patients who recovered have antibodies to the SARS-CoV-2 virus detectable in their blood. Most COVID-19 patients develop antibodies about 1-3 weeks after symptoms start. This is around the time when many patients start to recover. Patients who have had more severe disease appear to have higher levels of important neutralizing antibodies. Patients who had mild or asymptomatic COVID-19 have low levels of neutralizing antibodies (or even undetectable levels). In these persons it is possible the innate immune response and the $\mathrm{T}$ cell response cleared the virus. Recent studies have shown that neutralizing antibodies may disappear after 3 months ${ }^{41}$. Generally, a person who recovers from a viral infection is protected against new infection, if the antibodies are of adequate quality (neutralizing antibodies) and quantity (high levels). Changes in the virus sequence can make prior immunity less effective (eg. as happens with the influenza virus) • Protection from re-infection with the common cold caused by other milder coronaviruses is short-term (sometimes less than a year) For other coronaviruses, such as Severe Acute Respiratory Syndrome (SARS), antibodies have been detected a few years later. For COVID-19, we do not yet have enough data to confirm if antibodies protect, what antibody levels are required, or how long protection will last.

Herd immunity is the indirect protection of individuals from an infectious disease when a high proportion of a population is immune (usually through vaccination). Persons who haven't been infected, or who haven't had a good immune response, are protected because there are enough immune people around them to slow or stop person-to-person transmission. Many countries are testing for COVID-19 antibodies in the population or in specific groups. Fewer than 10\% of the general population ${ }^{42}$ have detectable COVID-19 antibodies (excepting in a few high intensity transmission sites or in specific small groups ${ }^{43}$. Worldwide most people remain susceptible to COVID-19 infection.

There is currently insufficient information to conclude whether people who have recovered from COVID-19 and have antibodies are protected from a second infection.

\section{How can we protect ourselves and others}

Stay home and self-isolate, Clean hands frequently, Avoid touching, Maintain a minimum physical distance of at least 1 meter from others, Stay away from crowds and stay away from 
poorly ventilated indoor spaces, Use a fabric mask where physical distancing of at least 1 meter is not possible, Use a medical / surgical mask if you may be at higher risk (age, medical conditions), Regularly clean and disinfect frequently-touched surfaces.

\section{Immunity- vaccination:}

Vaccines safely deliver an immunogen to the immune system to train it to recognize the pathogen when it is encountered naturally. The nature of the immunogen and route of administration affects the type of immune response induced, the safety, the protection and the duration of protection.

Immunogens: Attenuated pathogen (live, replicating, but not pathogenic),

Killed inactivated pathogen

Subunit (derived from pathogen)

Recombinant (viral antigen produced in a host cell and purified)

Peptides (synthetic fragments of antigen)

Vectored (viral pathogen expressed on a safe virus)

Nucleic acid (DNA or RNA coding for a viral protein and injected into body)

Route: Injection (in the muscle or under the skin), nasal spray, oral drops, aerosol.

\section{CONCLUSION:}

The development of vaccines is considered to be immunology's greatest success, as evidenced by the millions of lives saved. While immunologists have had great successes in the past, such as the elimination of smallpox and the near eradication of polio, many more complex diseases continue to challenge us. This technique generates whole proteome results quickly, providing scientists with the data to better understand the mechanisms behind successful immune response so that they can be translated into successful vaccines. It also provides a means to improve upon diagnostics in the clinic by generating a complete proteomic picture of a person's state of health. The faster we can refine our research methods and unveil protective immune mechanisms in humans, the better chance we stand at developing successful therapies and vaccines for the future.

Excessive inflammatory response with features of cytokine storm cause severe disease course and worsens the prognosis in COVID-19. Undoubtedly, drugs that directly target SARS-CoV2 would be the most effective treatments for COVID-19. There are hundreds of trials ongoing to find effective treatments for COVID-19 both targeting virus and consequent hyper inflammation including newly developed agents on phase studies or drugs that are approved for other indications. Boosting the immune system is a potential resource for the treatment of patients with COVID-19. The process and mechanism of the immune system can be a good 
source of knowledge for immune system development. Finally, nutrition (eg, dietary recommendations) to boost the immune system should be explored and recommended.

\section{REFERENCES:}

1. O'Shea J, Schwartz D, Villarino A, Gadina I, McInnes B, Laurence A. The JAK-STAT pathway: Impact on human disease and therapeutic intervention. Annu. Rev. Med.2015;66:311-328.

2. Zheng $\mathrm{C}$, Chen J, Chu F, Zhu J, Jin T. Inflammatory role of TLR-MyD88 signaling in multiple sclerosis. Front. Mol. Neurosci.2019; 12:314.

3. Varga J, Bui-Marinos M, Katzenback B.Frog skin innate immune defences: Sensing and surviving pathogens. Front.Immunol. 2018; 9:3128.

4. Kumar H, Kawai T, and Akira S. Pathogen recognition by the innate immune system. Int. Rev. Immunol. 2011;30(1):16-34.

5. Quaresma J. Organization of the skin immune system and compartmentalized immune responses in infectious diseases. Clin. Microbiol. Rev. 2019; 32(4).

6. Chaplin D. Overview of the immune response. J. Allergy Clin. Immunol.2010;125(2):S3-S23.

7. Agrawal B. Heterologous immunity: Role in natural and vaccine-induced resistance to infections. Frontiers in Immunology.2019; 10:2631.

8. Yu JC. Innate immunity of neonates and Infants. Frontiers in Immunology.2018;9:1759. 28. Fehr AH, Perlman S. Coronaviruses: an overview of their replication and pathogenesis. Methods Mol. Biol. 2015; 1282:1-23.

9. Lee KI, Wang CC, Lin MC, Kung CT, Lan KC, Lee CT. Effective strategies to prevent coronavirus disease-2019 (COVID-19) outbreak in hospital. The Journal of hospital infection. England; 2020.

10. Dong H. Characterization of an Immunodominant Epitope in the Endodomain of the Coronavirus Membrane Protein. Viruses. 2016;8(12):327.

11. Guo YR. The origin, transmission and clinical therapies on coronavirus disease 2019 (COVID-19) outbreak - An update on the status. Mil. Med. Res. 2020;7(1):11.

12. Weiss SR, Navas-Martin S. Coronavirus pathogenesis and the emerging pathogen severe acute respiratory syndrome coronavirus. Microbiol. Mol. Biol. Rev.2005;69(4):635-664.

13. Enjuanes L, Zuñiga S, Castaño-Rodriguez C, Gutierrez-Alvarez J, Canton J, Sola I. Molecular basis of coronavirus virulence and vaccine development. Adv. Virus Res.2016; 96:245-286. 
14. Song Z. From SARS to MERS, Thrusting coronaviruses into the spotlight. Viruses.2019;11(1).

15. Wang W. Detection of SARS-CoV-2 in different types of clinical specimens. JAMA; 2020.

16. Ebenso B, Out A. Can Nigeria contain the COVID-19 outbreak using lessons from recent epidemics?,” The Lancet. Global health. England; 2020.

17. Raoult D, Zumla A, Locatelli F, Ippolito G, Kroemer G. Coronavirus infections: Epidemiological, clinical and immunological features and hypotheses. Cell Stress; 2020.

18. D. Kesebir, M. Vazquez, C. Weibel et al., "Human boca virus infection in young children in the United States: molecular epidemiological profile and clinical characteristics of a newly emerging respiratory virus," Journal of Infectious Diseases, vol.194, no. 9, pp. 1276-1282, 2006.

19. L. van der Hoek, "Human coronaviruses: what do they cause?" Antiviral Therapy, vol. 12, no. 4 B, pp. 651-658, 2007.

20. J. Barenfanger, C. Drake, N. Leon, T. Mueller, and T. Troutt, "Clinical and financial benefits of rapid detection of respiratory viruses: an outcomes study," Journal of Clinical Microbiology, vol. 38, no. 8, pp. 2824-2828, 2000.

21. A. R. Falsey, Y. Murata, and E. E. Walsh, "Impact of rapid diagnosis on management of adults hospitalized with influenza," Archives of Internal Medicine, vol. 167, no. 4, pp.354-360, 2007.

22. D. E. Noyola and G. J. Demmler, "Effect of rapid diagnosis on management of influenza A infections,” Pediatric Infectious Disease Journal, vol. 19, no. 4, pp. 303$307,2000$.

23. Rothan HA, Byrareddy SN. The epidemiology and pathogenesis of coronavirus disease (COVID-19) outbreak. Journal of autoimmunity 2020:102433. doi: 10.1016/j.jaut.2020.102433

24. Zhou P, Yang X-L, Wang X-G, Hu B, Zhang L et al. Discovery of a novel coronavirus associated with the recent pneumonia outbreak in humans and its potential bat origin. BioRxiv 2020: 2020.2001.2022.914952. doi: 10.1101/2020.01.22.914952

25. Xu Z, Shi L, Wang Y, Zhang J, Huang L et al. Pathological findings of COVID-19 associated with acute respiratory distress syndrome. The Lancet Respiratory Medicine 2020. doi: 10.1016/s2213-2600(20)30076-X

26. Wang D, Hu B, Hu C, Zhu F, Liu X et al. Clinical characteristics of 138 hospitalized patients with 2019 novel coronavirus-infected pneumonia in Wuhan, China. Jama 2020. doi: 10.1001/jama.2020.1585 
27. Guan WJ, Ni ZY, Hu Y, Liang WH, Ou CQ et al. Clinical characteristics of coronavirus disease 2019 in China. The New England Journal of Medicine. 2020. doi: 10.1056/ NEJMoa2002032

28. Ivashkiv LB, Donlin LT. Regulation of type I interferon responses. Nature Reviews Immunology 2014; 14 (1): 36-49. doi: 10.1038/nri3581

29. Li G, Fan Y, Lai Y, Han T, Li Z et al. Coronavirus infections and immune responses. Journal of Medical Virology 2020; 92 (4): 424-432.

30. George MR. Hemophagocytic lymphohistiocytosis: review of etiologies and management. Journal of Blood Medicine 2014; 5: 69-86. doi: 10.2147/jbm.s46255

31. Ramos-Casals M, Brito-Zeron P, Lopez-Guillermo A, Khamashta MA, Bosch X. Adult haemophagocytic syndrome. Lancet (London, England) 2014; 383 (9927): 1503-1516. doi: 10.1016/s0140-6736(13)61048-x

32. McGonagle D, Sharif K, O'Regan A, Bridgewood C. Interleukin-6 use in COVID-19 pneumonia related macrophage activation syndrome. Autoimmunity Reviews 2020: 102537. doi: 10.1016/j.autrev.2020.102537

33. Sakaguchi S, Yamaguchi T, Nomura T, Ono M. Regulatory T cells and immune tolerance. Cell 2008; 133 (5): 775-787. doi: 10.1016/j.cell.2008.05.009

34. Sakaguchi S, Miyara M, Costantino CM, Hafler DA. FOXP3+ regulatory T cells in the human immune system. Nature Reviews Immunology 2010; 10 (7): 490-500. doi: $10.1038 /$ nri2785

35. Wang F, Nie J, Wang H, Zhao Q, Xiong Y et al. Characteristics of peripheral lymphocyte subset alteration in COVID-19 pneumonia. The Journal of Infectious Diseases 2020. doi: 10.1093/infdis/jiaa150

36. Wan S, Yi Q, Fan S, Lv J, Zhang X et al. Characteristics of lymphocyte subsets and cytokines in peripheral blood of 123 hospitalized patients with 2019 novel coronavirus pneumonia (NCP). MedRxiv 2020. doi: 10.1101/2020.02.10.20021832

37. COVID-19 CORONAVIRUS OUTBREAK, https:// www. worldometers. Info /coronavirus \& https://covidout.in/

38. Kumar NC, et al. Incidence of Dengue Fever in Febrile Patients and Co-Infection with Typhoid Fever in South India. Ann Med Health Sci Res. 2017; 7:111-113

39. Hobday RA Dancer S.J. Roles of sunlight and natural ventilation for controlling infection: historical and current perspectives. Journal of Hospital infection 2013, Vol. 84, ( 4) 271-282

40. https://www.nejm.org/doi/full/10.1056/NEJMc2025179 published 21 July 2020 Selected seroprevalence studies can be found here and here. 
41. Unity

Studies:

Early

Investigation

Protocols:

https://www.who.int/emergencies/diseases/novel-coronavirus-2019/technicalguidance/early-investigations

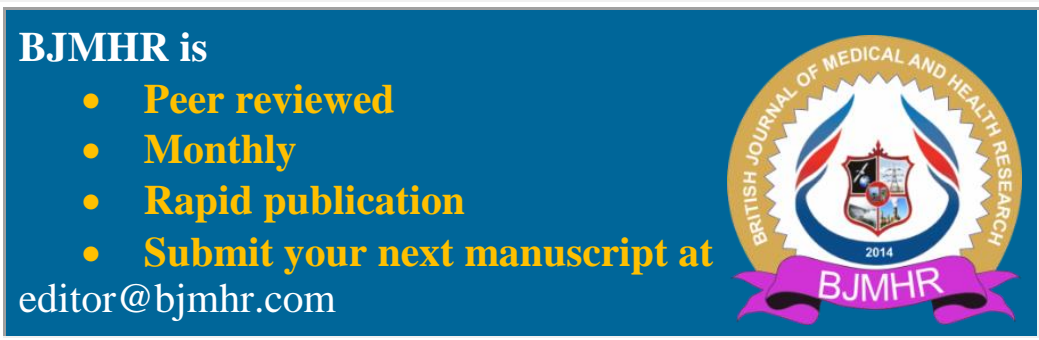

